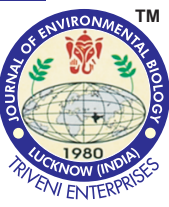

\title{
Identification of yeasts isolated from Chinese light cream in Inner Mongolia
}

\begin{tabular}{|c|c|c|}
\hline Paper received: 17. 04. 2018 & Revised received: 14. 11.2018 & Accepted: 03.01. 2019 \\
\hline
\end{tabular}

\section{Authors Info W. Ye ${ }^{1,2,3 *}$, J. Chen ${ }^{2}$, G. Huo ${ }^{2}$, P. Du' ${ }^{2}$, A. Li ${ }^{2}$ and L. Yang ${ }^{2}$ \\ ${ }^{1}$ College of Pharmacy, Shanghai University of Medicine \& Health Sciences, Shanghai, 200235 , China \\ ${ }^{2}$ Key Laboratory of Dairy Science, Ministry of Education, Northeast Agricultural University, Harbin, 150 030, China \\ ${ }^{3}$ Health School Affiliated to Shanghai University of Medicine \& Health Sciences, \\ Shanghai, 200 235, China \\ *Corresponding Author Email : 48472732@99.com}

\section{Edited by}

Prof. Muhammad Aqeel Ashraf

Reviewed by

Dr. H. Zhang

Dr. X. Jia

\section{Abstract}

Aim: This study was carried out to identify twenty-one yeast strains isolated from Chinese light cream in Inner Mongolia.

Methodology: The morphological, physico-chemical characterization of yeast strains were performed. ID $32 \mathrm{C}$ system was used to examine the carbohydrate fermentation. The $26 \mathrm{~S}$ rDNA sequencing of yeasts were amplified by the primers (26SrDNA1: 5' GCA TAT CAA TAA GCG GAG GAAAAG 3'; 26SrDNA2: 5' GGT CCGTGTTTCAAGACG G3').

Results: The phylogenetic tree was constructed. According to the results of BLAST in NCBI, twenty-one yeasts were identified as Kluyveromyces marxianus (9), Pichia kudriavzevii (8), Saccharomyces cerevisiae (3) and Kluyveromyces lactis (1) according to 26S rDNA representative amplicons sequencing. Kluyveromyces marxianus and Pichia kudriavzevii were the main yeast strains present in light cream.

Interpretation: The Chinese light cream is a traditional milk product popular in Mongolia. Some researchers pay more attention on the lactic acid bacteria, but few reports on the yeast in it. In traditional fermentation method, yeast is also contributed to the flavor of light cream. So we identified the yeast of it.

Key words : 26S rDNA, Light cream, Inner Mongolia, Yeast

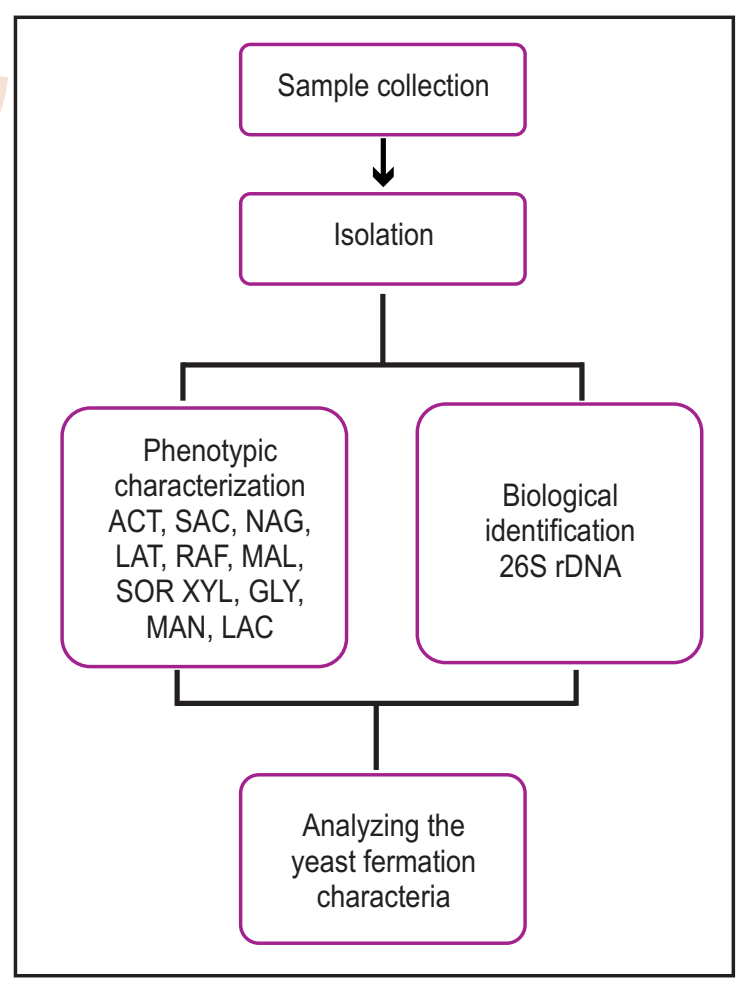

Citation: Ye, W., J. Chen, G. Huo, P. Du, A. Li and L. Yang: Identification of yeasts isolated from Chinese light cream in Inner Mongolia. J. Environ. Biol., 40, 468-471 (2019). 


\section{Introduction}

Light cream is a popular traditional milk product in Mongolia and Xinjiang, China. Dairy products such as light cream and sour milk are usually made by traditional fermentation methods at home of Inn Mongolia. Milk is usually used to make sour milk, light cream, milk wine and other dairy products.

Recently, many yeasts have been isolated from different fermented food, such as Koumiss and cachaca (Padilla et al., 2016; Mu et al., 2012; Meririana et al., 2009; Xiao and Yuqing, 2018). Kluyveromyces lactis and Torulaspora delbrueckii have been isolated from the canastra cheese production (Rafaela Pereira Andrade et al., 2017; Sedaghathoor and Shahrajil, 2018). In Thailand, few Candida and Pichia strains have been isolated from yeast starter doughs for Chinese steamed buns (Naphatrapi, et al., 2009; Zhao and Li, 2018). Most yeasts are isolated from wine (Barrajón et al., 2009; Gonza'lez, et al., 2007; Rosalinda et al., 2010; Mohammed, 2018). Recently, from dairy products have been isolated and identified Kluyveromyces marxianus, Pichia fermentans, Pichia cactophila, Candia zylanoieds were isolated form Koumiss in Inner Mongolia and Xinjiang (Xiaoxu et al, 2016; Sultana et al., 2018). With the development of genomics and bioinformatics, the classification of yeast is not only based on their morphological characteristics and physiological and biochemical characteristics. The $26 \mathrm{~S}$ sequence analysis method is commonly used for the identification of yeast, and it can be more accurate to analyze the distribution of yeast strains (Visintin et al., 2016; Brilhante et al., 2016; Ying et al., 2018).
We analyzed some lactic acid bacteria from traditional Chinese fermented food before. Some yeasts were isolated from the the Chinese light cream. We thought the yeasts maybe also give reference good taste and smear performance. So in this paper we analyzed their variety.

\section{Materials and Methods}

Sample collection: Twenty-one samples were collected from traditional light cream which were made by traditional fermentation methods in Inner Mongolia. The light cream was collected into a sterilized centrifuge tube to send to the Key Lab of Dairy Science after sealing well. All the samples were kept at $20^{\circ} \mathrm{C}$ for isolation

Isolation of yeasts: Ten gram of each sample was resuspended in $90 \mathrm{ml}$ of sterile $\mathrm{NaCl}$ solution (0.9\%) and homogenized three times. The suspension was diluted tenfold in $\mathrm{NaCl}$ solution $(0.9 \%)$, and $100 \mu$ lilution were spread on the surface of YPD plates to incubate at $28^{\circ} \mathrm{C}$ for five days. The appearance of colony was observed, single colonies of yeasts were purified by streak plating on the YPD agar (1\%yeast extract, 2\% peptone, $2 \%$ glucose and $2 \%$ agar).

Phenotypic characterization: Purified yeast were inoculated into YPD broth separately and incubated at $28^{\circ} \mathrm{C}$ for five days. ID $32 \mathrm{C}$ tests were used for investigating the physico-chemical characterization of yeast by reacting with different sugars (BioMérieux, USA). The isolaties were inoculated into suspension medium, 250 $\mu$ l mixed medium was extracted into C Medium of ID $32 \mathrm{C}$ test box, then $135 \mu \mathrm{l}$ mixture medium was added into each

Table 1 : Identification carbohydrate fermentation of yeast (+means it could ferment this carbohydrate)

\begin{tabular}{|c|c|c|c|c|c|c|c|c|c|c|c|c|c|}
\hline & GAL & ACT & SAC & NAG & LAT & RAF & MAL & SOR & XYL & GLY & MAN & LAC & GLU \\
\hline S-1 & & & & + & + & & & & & + & & & + \\
\hline S-2 & + & + & + & - & + & + & 0 & + & $?$ & 0 & + & + & + \\
\hline S-3 & + & + & + & 0 & + & + & 0 & + & + & & + & + & + \\
\hline S-4 & + & + & + & 0 & + & + & 0 & + & + & 0 & 0 & + & + \\
\hline S-5 & 0 & 0 & 0 & 0 & + & 0 & 0 & 0 & 0 & & 0 & 0 & + \\
\hline S-6 & + & + & + & 0 & + & + & 0 & + & & 0 & + & + & + \\
\hline S-7 & & 0 & 0 & + & + & 0 & 0 & & 0 & & 0 & 0 & + \\
\hline S-8 & & + & + & 0 & + & + & 0 & + & & + & + & + & + \\
\hline S-9 & 0 & 0 & 0 & 0 & + & 0 & 0 & + & & + & & 0 & + \\
\hline S-10 & + & + & + & 0 & + & + & 0 & + & & & & + & + \\
\hline S-11 & + & + & + & 0 & + & + & 0 & + & + & & + & + & + \\
\hline S-12 & + & + & + & 0 & + & + & 0 & + & & & + & + & + \\
\hline S-13 & + & 0 & + & 0 & 0 & + & + & 0 & 0 & 0 & 0 & 0 & + \\
\hline S-14 & & & & & + & & & & & + & & & + \\
\hline S-15 & + & + & + & 0 & + & + & 0 & + & + & & & + & + \\
\hline S-16 & + & + & + & 0 & + & + & 0 & + & & 0 & + & + & + \\
\hline S-17 & + & 0 & & 0 & & & + & 0 & 0 & 0 & 0 & 0 & + \\
\hline S-18 & + & + & + & 0 & + & + & 0 & + & + & 0 & + & + & + \\
\hline S-19 & + & + & + & 0 & + & & 0 & & & + & & + & + \\
\hline S-20 & + & 0 & 0 & + & + & 0 & 0 & + & & & & 0 & + \\
\hline S-21 & + & & & & + & & + & & & & & & + \\
\hline
\end{tabular}




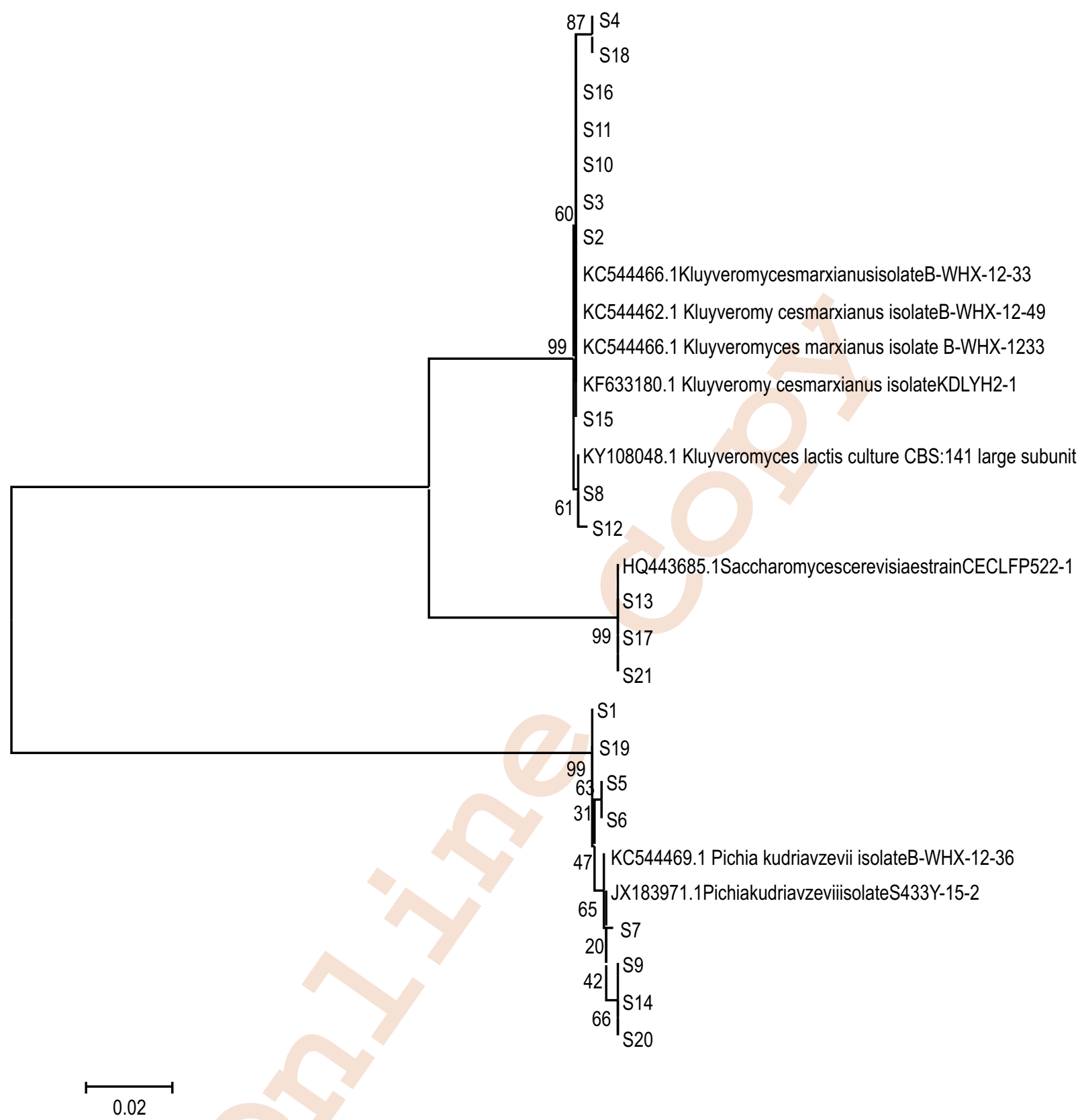

Fig. 1 : Phylogenetic tree based on 26S rRNA D1/D2 domain sequence alignment. All bootstrap percentages over 50\% are shown.

cupule of ID $32 \mathrm{C}$ incubated for $24-48 \mathrm{hrs}$ at $30^{\circ} \mathrm{C}$. The reaction results were identified by ATB machine.

Identification of yeasts: The D1/D2 domains of 26S rDNA encoding gene were amplified by the primers (26SrDNA1: 5 ' GCA TAT CAA TAA GCG GAG GAAAAG 3'; 26SrDNA2: 5' GGT CCG TGT TTCAAG ACG G 3') (Fell et al. 2000). Amplified PCR of 265 rDNA procucts were purified. The sequence was tested by Sangon Biotech (Shanghai). The sequences obtained were aligned with known $26 \mathrm{~S}$ rDNA gene sequences procured from the Genbank by BLAST. The generated percent homology scores were used for the identification of yeast isolations. The sequences were aligned, and phylogenetic tree was constructed by neighbor joining method using Mega Software (version 5.0).

\section{Results and Discussion}

ID32C is the most commonly used identification system for yeast. It can identify the use of yeast for different carbohydrates, with high accuracy, but limited identification. Therefore, it is necessary to identify it with 26S (Fell et al., 2000). The examination of carbohydrate fermentation were tested by the 
Table 2 : Identification of $26 \mathrm{~S}$ rDNA of yeast isolates

\begin{tabular}{lll}
\hline Strain & Identification & Homology\% \\
\hline S-1-5-6-7-9-14-19-20 & Pichiakudriavzevii & $99-100$ \\
S-2-3-4-10-11-12-15-16-18 & Kluyveromyces marxianus & 100 \\
S-8 & Kluyveromyces lactis & 99 \\
S-13-17-21 & Saccharomyces cerevisiae & 99 \\
\hline
\end{tabular}

ID 32C system. The main phenotypic characteristics of isolates are summarized in Table 1. All strains can ferment glucose. The differences of fermentation sugars among strains were observed for ACT, SAC, NAG, LAT, RAF, MAL, SOR, XYL, GLY, MAN, LAC. The substrates of Gal, ACT, SAC, NAG, LAT, RAF, MAL, SOR, $X Y L, G L Y, M A N$ and LAC are galactosum, D- galactose, actidione, saccharose, acetylglucosamine, lactic acid, Draffinose, D-maltose, D-sorbitol, D-xylose, , D- mannitol and Dlactose.

The 26S rDNA of isolated yeasts were determined by the nucleotide sequence of D1/D2 domains. According to BLAST results, eight strains isolated S-1, S-5, S-6, S-7, S-9, S-14, S-19, S-20 were similar to Issatchenkia orientalis, nine strains S-2, S-3, S-4, S-10, S-11, S-12, S-15, S-16, S-18 were similar to Kluyveromyces marxianus, three strains S-13, S-17, S-21 were similar to Saccharomyces cerevisiae. One strain S-8 was similar to Kluyveromyces lactis (Table 2). The phylogenetic tree showed the maximum identity of each strain which was similar to the Blast results (Fig.1). Except S-12, which was more similar to Kluyveromyces marxianus than Kluyveromyces lactis when BLAST in NCBI, however in the phylogenetic tree it was closer to S-8 which belong to Kluyveromyces lactis.

In this study, The yeast isolated from Inner Mongolia in china, and all isolates were identified based on physiological characteristics and analysis of $26 \mathrm{~S}$ rDNA sequences. Most of the yeast can fermented Lactose excepted S13 and S17, but S13 and S17 can fermented maltose which means these two strains can fermented the milk well with other strains. Kluyveromyces marxianus and Pichia kudriavzeviiare the major yeasts isolated from the dilute cream. Kluyveromyces marxianus can improve the volatile flavor components in the fermentation of dilute cream (Wang et al., 2015). More experiment will be done in the future.

\section{References}

Andradea, R.P., C.N. Melo, Z. Genisheva, R.F. Schwan and W.F. Duartea: Yeasts from Canastra cheese production process: Isolation and evaluation of their potential for cheese whey fermentation. Food Res. Inter., 9, 72-79(2017).

Barrajón, N., M. Arévalo-Villena, L.J. Rodríguez-Aragón and A. Briones: Ecological study of wine yeast in inoculated vats from La Mancha region. Food Control, 20, 778-783(2009).

Brilhante, R.S., J.E. Maia-Júnior, J.S. Oliveira, G.M. Guedes, A.L. Silva, F.B. Moura, J.A. Sales, D.S. Castelo-Branco, J.J. Sidrim, R.A.
Cordeiro and W.A. Pereira-Neto: Yeasts from the microbiota of bats: A focus on the identification and antimicrobial susceptibility of cryptic species of Candida. J. Med. Microbiol., 65, 1225-1228 (2016).

Fell, J.W., T. Boekhout, A. Fonseca, G. Scorzetti and A. Statzell-Tallman: Biodiversity and systematics of basidiom ycetous yeasts as determined by large-subunit rDNA D1/D2 domain sequence analysis. Int. J. Syst. Evol. Microbiol., 50, 1351-1371 (2000).

García-Fernández, D., B. González, I. Izidoro, B. Esteve-Zarzoso, G. Beltran and A. Mas: Yeast biodiversity from DOQ Priorat Uninoculated fermentations. Front. Microbiol., 15, 930(2016).

Gonza'lez, S.S., E. Barrio and A. Querol: Molecular identification and characterization of wine yeasts isolated from Tenerife (Canary Island, Spain). J. App. Microbiol., 102, 1018-1025 (2007).

Luangsakul, N., S. Keeratipibul, S. Jindamorakot and S. Tanasupawat: Lactic acid bacteria and yeasts isolated from the starter doughs for Chinese steamed buns in Thailand. LWT - Food Sci. Technol., 42, 1404-1412(2009).

Mohammed, G.E.A.T.: Productivity of pure stands and intercropped forage Sorghum and Hyacinth bean. Malays. J. Sustain. Agric., 2 , 05-06(2018).

$\mathrm{Mu}, \mathrm{Z}$., X. Yang and H. Yuan: Detection and identification of wild yeast in Koumiss. Food Microbiol., 31, 301-8(2012).

Nova, M.X.V., A.R.P. Schuler, B.T.R.V. Brasileiro and M.A. Morais: Yeast species involved in artisanal cachaça fermentation in three stills with different technological levels in Pernambuco, Brazil. Food Microbiology, 26, 460-466 (2009).

Padilla, B. and M.F. Rocha: Yeasts from the microbiota of bats: A focus on the identification and antimicrobial susceptibility of cryptic species of Candida. J. Med. Microbiol., 65, 1225-1228(2016).

Sedaghathoor, S. and H.R. Shahrajil: Unexpected rooted in shoot-tip cuttings of ponytail palm (Beaucarnea recurvata). Malays. J. Sustain. Agric., 2, 24(2018).

Sultana, T., S. Rahman, N. Naher, R.M. Masum, A.H.A. Ahmed and R. Islam: Performance of fruit vegetables in summer under mahagony based agroforestry systems. Malays. J. Halal Res., 1, 08-14 (2018).

Urso, R., K. Rantsiou, P. Dolci, L. Rolle, G. Comi and L. Cocolin: Yeast biodiversity and dynamics during sweet wine production as determined by molecula rmethods. FEMS Yeast Res., 8, 10531062 (2010).

Visintin, S., V. Alessandria, A. Valente, P. Dolci and L. Cocolin: Molecular identification and physiological characterization of yeasts, lactic acid bacteria and acetic acid bacteria isolated from heap and box cocoa bean fermentations in West Africa. Int. J. Food Microbiol, 216, 69-78 (2016).

Xiao, D. and L. Yuqing: The evaluation indexes of scientific and technological achievements in Jiangxi Province, China. Acta Sci. Malays., 2, 21-22 (2018).

Xiaoxu, Z., G. Wupeng, L. Xiuzhen, L. Xiaopeng, S. Yaxin, M. Junying, F. Ruoyu and G. Wei: Diversity and fermentation characteristics of yeasts in Koumiss collected from Inner Mongolia and Xinjiang. Food Sci., 37, 156-161(2012).

Ying, H.S., M.S. Anuar and M.Z. Mohd Nor: Drying, colour and sensory characteristics of 'Berangan' Banana (Musa accuminata) flesh dried using a microwave oven. Malays. J. Halal Res., 1, 10-14 (2018).

Zhao, J. and S.M. Li: The impact of tourism development on the environment in China. Acta Sci. Malays., 2, $01-04$ (2018). 